\title{
A discussion of the cognitive load in collaborative problem-solving
}

\section{The decision-making phase}

\author{
Gwendolyn Kolfschoten • Simon French • \\ Frances Brazier
}

Received: 28 July 2013/ Accepted: 23 September 2014/Published online: 25 October 2014

(C) Springer-Verlag Berlin Heidelberg and EURO - The Association of European Operational Research Societies 2014

\begin{abstract}
Collaborative problem-solving is inherent to complex participatory systems. Supporting collaborative problem-solving is challenging. Problem-solving, like all knowledge-based collaborative effort, requires significant cognitive effort. Understanding the cognitive load involved is important for the design of group support techniques and tools. This paper presents results on cognitive load related to problem-solving in the decision-making phase, in which alternative solutions or options are evaluated and the group engages in, inter alia, consensus building, negotiation or commitment building to agree on a course of action. This decisionmaking phase is less often studied than its preceding phases, i.e. divergence or brainstorming of alternatives, and convergence of alternatives to a parsimonious set to consider for the final phase of decision-making. The cognitive activities involved in these two phases precede the cognitive activities involved in decision-making. This paper focuses on managing cognitive load involved in the decision-making phase based on the results of literature analysis, an overview of techniques and finally expert validation. The paper concludes with an outlook of classes of cognitive tasks in collaborative problem-solving and the implications of this perspective.
\end{abstract}

Keywords Collaborative problem-solving $\cdot$ Decision-making $\cdot$ Cognitive load

Mathematics subject classification $90 \mathrm{Bxx} \cdot 91 \mathrm{Cxx}$

G. Kolfschoten $(\varangle) \cdot$ F. Brazier

Delft University of Technology, Delft, The Netherlands

e-mail: G.L.Kolfschoten@tudelft.nl

S. French

Department of Statistics, University of Warwick, Coventry, UK

e-mail: simon.french@warwick.ac.uk 


\section{Introduction}

Collaborative problem-solving is a sine qua non for innovation and productivity of organizations (Frost and Sullivan 2007). One of the approaches to problem-solving found in Operations Research is described as Problem Structuring. This facilitated approach to problem-solving is participative with the objective of creating commitment for the implementation of results (Franco and Montibeller 2010). The focus of the paper will be on managing cognitive load as the problem structuring phase in problem-solving moves into the final phase of decision and implementation where building commitment and agreement are critical.

Problem structuring methods are most often applied in the context of 'messy' or 'wicked' problems. These problems are characterized by many stakeholders, a complex problem, uncertainty, and interference with other systems or problems. Examples of these methods are Soft Systems Methodology, Strategic Options Development and Analysis and Strategic Choice. Eden and Ackermann (2006) and Rosenhead (2006) list their similarities or characteristics as:

1. The use of a model as a transactional object.

2. The objective of increasing the overall productivity of the group process.

3. Attention to facilitation of an effective participative group process.

4. The significance of facilitation skills in effective model building.

5. A focus on creating agreement and closure rather than solutions.

6. A respect for multiple perspectives.

7. An alternation between analysis of judgemental inputs and the application of judgement of analytic outputs.

Facilitated problem structuring has the objective of supporting a group of stakeholders in reaching an agreement built on shared understanding. It does this by introducing one or more structures which provide simplified but informative common perspectives on the problem, thus helping 'manage' information overload that can accrue in complex, multi-stakeholder wicked problems. An important purpose of problem structuring is to manage and balance cognitive load.

Cognitive load is the cognitive effort made by a person to perform a task (Sweller et al. 1998). Individual cognitive load in a task is typically higher for collaborative tasks than for individual tasks (Dillenbourg and Betrancourt 2006), as they involve not only information processing, but also interaction and communication tasks. Therefore, groups often benefit from tools and facilitation to focus their cognitive effort (Niederman et al. 2008). Particularly in problem structuring tasks, an overload of ideas can be created as multiple perspectives are explored (Franco and Montibeller 2010). To understand which tools and techniques are effective in supporting collaborative problem structuring effort, it is crucial to understand their impact on cognitive load. For this, cognitive processes constituting this cognitive load need to be untangled.

Problem-solving is described as a process with several iterative phases including problem formulation, analysis, finding/designing solutions, analyzing solutions, choice and implementation and various additional evaluation and verification steps 
(Ackoff 1978; Brady 1967; Checkland 1981; Couger 1995; Drucker 1967; Hackman and Morris 1975; Mitroff et al. 1974; Simon 1960, 1973; Vennix et al. 1992). From a cognitive perspective, some of these tasks are similar. To this purpose three distinct cognitive phases are distinguished:

- First, brainstorming (also called divergence) to gather, create share or brainstorm information and identify alternatives for decision-making.

- Second, analysis and structuration of the information available to create meaning and shared understanding (also called convergence).

- Third, decision-making to reach common ground and agreement based on the information analyzed.

This paper summarizes the cognitive processes in the first two phases, published in previous work (Kolfschoten 2011; Kolfschoten and Brazier 2012), and then focuses on the third phase, decision-making to reach common ground and agreement in which characteristics 5, 6, and 7 above are critical. In this phase, decision makers from different perspectives judge alternative solutions to build agreement on a solution they can jointly implement. Techniques such as SODA and JOURNEY (Eden and Ackermann 2004) describe this as a final phase involving negotiation and building agreement.

Knowledge-intensive collaborative problem-solving tasks such as decisionmaking often require high cognitive effort to consider alternatives, their implications, their value, and the group members' personal preferences for these. Further, collaborative decision-making requires a team to perform a task jointly, thus requiring interaction and coordination of cognitive effort (Dillenbourg and Betrancourt 2006). Coordination and support of collaboration can be offered by a group member (leader) or by an external facilitator or mediator, who can help the group to focus cognitive effort and offer tools and techniques to support effective cognitive effort. Furthermore, techniques and tools such as decision and negotiation support systems, and sometimes also training, can offer guidance in collaborative activities (Dennis and Wixom 2002) and in decision-making particularly (Balthazard et al. 1998; Pervan 1998; Wheeler and Valacich 1996).

In the decision-making phase the group judges the value of alternatives, compares them, and considers both rational and strategic implications to then agree on a course of action. Based on the literature and examples of existing collaboration support techniques and tools, this paper explores the role and design implications of cognitive load in the decision-making phase of collaborative problem-solving tasks. This paper focuses primarily on the cognitive activities in the decision-making phase, validated by a limited number of experts. The paper concludes with a discussion on the impact of cognitive load on the effectiveness of problem structuring methods.

\section{Cognitive load}

Cognitive load theory (CLT) distinguishes various design principles to use cognitive capacity efficiently and effectively in the context of learning (Sweller et al. 1998). 
However, in the context of collaborative problem-solving, little research has been devoted to understanding the cognitive implications of process and technology design. Collaborative problem-solving, in particular decision-making, requires some form of learning, specifically to create shared understanding about alternatives and their implications, as well as each other's perceptions, beliefs and preferences towards these alternatives. As such, the learning domain provides valuable insights to support problem-solving tasks. In decision-making, this cognitive complexity is extensively studied in psychology (Slovic et al. 1977) and in the design of, e.g. expert systems, artificial intelligence (Simon 1980) and decision support systems (Klein and Hirschheim 1985). While there exist cognitive models of decisionmaking, these mostly focus on individual decision-making rather than group decision-making (French 2007). Also, these models do not consider the different sources of complexity that require cognitive effort, and are triggered in a collaborative decision-making process.

Cognitive load can be defined as the cognitive effort made by a person to understand and perform his/her task (Sweller 1988). Cognitive load has both a taskbased dimension (mental load) and a person-based dimension (mental effort) (F.G.W.C. Paas and van Merrienboer 1994; Sweller et al. 1998). This paper proposes a third dimension of cognitive load in mutual coordination of effort in a group task. Dillenbourg and Betrancourt call this collaboration load (Dillenbourg and Betrancourt 2006). Task-based cognitive load has a further perceptual and cognitive dimension, related to the amount of information presented, and the amount that needs to be processed in working memory to accomplish the task (Fitousi and Wenger 2011). A similar effect can be considered in collaboration load, where participants consider the directly observed actions of others and the perceived 'intentions' or stakes behind those actions to understand the motivation of their fellow group members, and ultimately to predict their behavior. The concept of cognitive load in CLT is associated with 'computer-based' cognitive model in which the main 'processor' is represented by our short-term or working memory, also called central executive, which is limited in capacity (Farrington 2011; Miller 1956; Simon 1974). Problem-solving tasks are mainly associated with the prefrontal cortex, which is also used to recall things from memory and to inhibit distraction (Rock 2009). In CLT, the complex cognitive activities of these brain parts are simplified as a limited resource for information processing. While cognitive- and neuro-psychologists offer ample debate on the different ways in which cognitive tasks are performed in the brain, they agree that our capacity to process information is limited, and that these limitations are actively experienced in problem-solving tasks (Rock 2009). Furthermore, research in instructional design has provided ample evidence that the manner in which information is offered and structured has significant effects on performance in problem-solving (Sweller 1988). In problem structuring methods, a similar effect is intended, when problem structuring helps the group to balance cognitive attention to enable them to consider the multiperspective complexity of the problem in a more effective way.

Problem-solving is a complex task, and can benefit from support. Early research from Simon already indicated that one can learn a lot about problem-solving and how to support it, when looking at it from a cognitive perspective (Simon 1980). 
CLT assumes people have a short-term or working memory for information processing, and a long-term memory to store information (Sweller 1988). Information in our long-term memory is related, networked or associated through schema (Rock 2009). In a process called automation, also called chunking (Miller 1956; Simon 1974), more complex information is processed in our working memory by structuring or grouping it in these schemas. These schemas allow faster access to the information stored in long-term memory, and processing more (schematized) information in working memory. With limited cognitive capacity, cognitive overload is easily reached in complex cognitive tasks such as problem-solving and decision-making considered in this paper. Cognitive overload can impair performance and decision-making, create stress, cause difficulty in retrieving knowledge, impede creativity, and cause difficulty in analyzing and organizing knowledge, and impede schema building and learning (Eppler and Mengis 2004). Overload can be handled through structuration, organizing and coordinating, and through the use of information systems that help filter information (Eppler and Mengis 2004). Cognitive overload creates ineffectiveness, but too low cognitive load can also be ineffective as people loose attention for the task. When cognitive load is at a right level, participants can get in a state of flow (Czikszentmihalyi 1990). The cognitive load of a task differs for each individual, depending on their experience in the domain and skill in the type of problem-solving task (Kalyuga et al. 2003; Kolfschoten et al. 2010). Furthermore, people can become distracted while performing a task or use ineffective ways of processing the information due to fatigue or lack of skill (Rock 2009).

CLT in the context of learning explains how cognitive capacity is used to construct schema and use schema for problem-solving tasks. Three types of cognitive load (Sweller 1988) are distinguished:

- Intrinsic cognitive load is the cognitive load that is inherent to the task, defined by the intrinsic task complexity, but personal, depending on the experience level of the person performing the task.

- Extraneous cognitive load is the cognitive load caused by the presentation and transition method of the information. Extraneous load should be reduced as much as possible, as it is ineffective. However, it cannot be completely eliminated. Extraneous load also covers collaboration load, the mental effort required to coordinate effort.

- Germane cognitive load is the cognitive load instrumental to building schemata and storing them in the long-term memory. For learning, germane load should be stimulated to focus attention not just on processing information and principles, but on storing them in long-term memory.

Limiting unproductive mental activity, and focusing collaborators on effective cognitive effort is a critical challenge for information systems design (Bray 2008), particularly systems that support collaboration and collaborative effort. Information systems are socio-technical systems, a combination of technology, people and processes. In these systems, technology and process structuration support decision- 
making by reducing the complexity of information. CLT provides different methods to reduce extraneous (Sweller et al. 1998) and intrinsic cognitive load (Pollock et al. 2002), and to focus effort on germane load (Paas et al. 2004). With these approaches, CLT has provided new insights in instructional design improving learning efficiency and effectiveness. In collaboration, cognitive load has many sources. It can originate from the information shared among participants through various communication channels, from constructing and thinking up new information, from explaining or arguing positions, from assessing value, implications and effects of decisions, from various procedures, and from distractions. While collaborative problem-solving and decision-making is different in many ways from learning tasks, it also bears similarities. Elementary cognitive activities such as understanding, deciding, recalling (from memory), memorizing (store in memory) and inhibiting (to push away distracting thoughts or stimuli) (Rock 2009) are the cornerstones of both the problem-solving and the learning process. The assumption on which this paper is based is that this cognitive perspective will provide new insights in the effectiveness and efficiency of collaborative problem structuring.

\section{Previous work}

As described above, the elementary cognitive process of problem structuration is grouped in roughly three phases: a divergent phase, a convergent phase and a 'decision-making' phase.

Divergence, also called generation or brainstorming, (Briggs et al. 2009) often produces a large volume of content of varying relevance, across multiple levels of abstraction and of varying granularity. This knowledge, shared and created by a group, needs to be summarized in a manageable way to create an overview that can be used for further analysis, evaluation or decision-making. In the decision-making phase the focus is on building agreement, where in convergence, the focus is on organizing ideas, creating shared understanding and developing a set of detailed alternatives with consistency and completeness. Convergence is not about choosing or judging alternatives, only about structuring and refining them.

Brainstorming and convergence are the focus of previous work (Kolfschoten 2011; Kolfschoten and Brazier 2012). These papers describe brainstorming activities as they occur during a facilitated divergence process with seven subphases:

1. Preparation, a phase in which the task and objective become clear, as well as the way this task will be executed collaboratively. Further, there is a transition from understanding the tasks and actually starting the brainstorm.

2. Brainstorming itself starts with ideation; conceiving new ideas through the exploration of different perspectives on the problem.

3. Once ideas are conceived there are various considerations to the quality, fit and acceptance of the idea.

4. Based on a decision to share the idea, it needs to be explicated to actually share it with the group. 
5. Participants then need to listen to or read the ideas of others, and reflect on these ideas which can spark new ideas, but can also trigger further consideration of implications of these ideas.

6. Besides reflection on single ideas, participants can also reflect on the overall set of ideas to consider, e.g. quality and completeness.

7. Finally, participants can get distracted in the process of ideation.

Naturally, this process is highly iterative, and all types of reflection will lead to new, combined or improved ideas (Stroebe et al. 2010). While brainstorming is often used in practice, and techniques for brainstorming are available in a wide variety, their effectiveness has been the discussion of many scientific publications (Nijstad et al. 2006; Pinsonneault et al. 1999; Reinig et al. 2007; Santanen 2005; Valacich et al. 1994). In problem structuring, brainstorming or divergence has two key objectives: to identify creative ideas (solutions/alternatives), and to encourage mutual learning (sharing different perspectives).

After ideation, a group can have many ideas, and depending on the way they shared these ideas, and the history of the group, there is an initial level of shared understanding. However, often, even in groups with high common ground and background, some ideas need explanation. Furthermore, it can be important to structure the ideas to create overview and to reduce or filter them to work towards a more parsimonious set of alternatives that can be used for comparison and decisionmaking. This process again can be described in six sub-phases.

1. Preparation, similar to the preparation for the brainstorming task.

2. Analysis of the ideas is the first step, participants should read or listen to these ideas and identify where they see misunderstanding, inconsistency, overlap, etc.

3. Next the actual convergence process takes place. This is a process of abstraction, comparison, re-formulation and selection or filtering. The ideas are placed in a structure and polished to be distinguishable and consistently formulated to meet criteria for completeness and quality.

4. During this convergence phase, again participants will reflect on the implication and effects of the structuration process.

5. Besides reflection on single ideas, there is also in this process an overall reflection in which consistency, quality and completeness of the set of ideas in answer to the problem is considered.

6. Finally, also in this process, people get distracted.

After the main phases of divergence and convergence, the problem-solving process focuses on comparing alternatives and selecting the alternative that best solves the actual problem. ${ }^{1}$ This third decision-making phase seems to be the most substantial phase in problem-solving. In practice, often groups consider only known alternatives, and do not engage in out-of-the-box brainstorming, which also

\footnotetext{
${ }^{1}$ This paper refers to this phase as the decision making phase. The authors realize that the term decision making often refers to the entire problem solving process.
} 
reduces the need for an extensive convergent phase. However, the decisionmaking phase, where alternatives are compared and a choice is made, seems likely to occur in all problem-solving tasks where multiple stakeholders are involved. While all three phases are critical and need support, this paper focuses on the decision-making phase, where problem structuring methods are aimed to help the group in building agreement and at the same to ensure an analytical judgment based on outcomes as described in characteristic 5 and 7 of the problem structuring methods above.

\section{Decision-making in problem-solving}

When groups join their forces to solve a problem, they need to make decisions that represent an agreement in the group. Even in situations where one group member has the power to make decisions alone, it often makes sense to involve others in the decision-making process to use their expertise, and more importantly to get buy-in or support for the results. Thus, group decision-making requires consensus building and negotiation to establish trust in buy-in and support.

Decision-making typically can be described along two dimensions: one represents the rational choice aspect, which reflects on the objective qualities of the alternatives. However, alternatives of high quality are not necessarily the ones that most people want given their stakes, personal goals and priorities and the other is the social dimension, the extent to which there is commitment to the decision outcome. Groups can decide to use a very simple decision principle (we choose what the majority wants or judge based on the average score), or they can engage in conflict resolution to unravel their disagreements and find a solution that has full support of all members. In all scenarios, participants need to understand the alternatives, to judge these alternatives and determine their value, and to consider the impact of the choice on their personal or strategic goals. The level of complexity of the decision-making task depends on the number of alternatives a group is willing to consider. A group can choose to focus on comparing the value of a few alternatives to maximize value instead of choosing to consider new alternatives. Furthermore, conflicting objectives may need to be traded off, for instance costs and benefits, or when there is risk or uncertainty related to the alternative. These objectives need to be considered and impact the overall evaluation of ideas and complexity of the decision-making task. Decision support can be used to consider these different evaluation criteria and their relative importance to support evaluation and the identification of the source of disagreement.

Several approaches to decision-making are compared by (Schwenk 1984). This paper adapts this approach for group decision-making. Note that for the sake of explanation this process is described linearly. In practice the process is not linear. Most often groups iterate and alternate between phases. Figure 1 visualizes the decision-making process as a linear process with six phases, similar to the phases described for brainstorming and convergence above. 
4.1 Phase 1: decision preparation

This phase prepares two aspects:

(A) Selecting the criteria for evaluation. (Note that identifying and agreeing on a set of criteria can be a process that includes generating possible criteria, converging to a smaller set and decision-making to choose appropriate criteria. The focus of this paper is on the decision-making phase).

(B) Establishing the rules for decision-making. (The choice of rules for decisionmaking is often a decision process in itself, and as such is outside the scope of the decision-making phase, which is addressed in this paper).

\subsection{Phase 2: evaluation}

This phase assesses, evaluates and/or compares the set of alternatives that is created in the convergence phase. When all decision makers have shared their assessment and preferences, these assessments and values are combined to provide an overview of the group's assessment and preferences, and the level of agreement on the evaluation.

\subsection{Phase 3: aggregation}

In this phase, the individual evaluations are aggregated to the group level to be compared. Based on this first overview of evaluation results, disagreements are explored to see if some results are based on misunderstanding.

\subsection{Phase 4: resolving misunderstanding}

In this phase, disagreements based on misunderstanding are resolved through information sharing, and explanations of differences in perception. When these misunderstandings are resolved, differences in preferences remain.

\subsection{Phase 5: negotiation}

In this phase, negotiation is used to build support towards the decision that helps to resolve the problem or offer a solution to overcome different perspectives.

\subsection{Phase 6: confirmation}

Finally the phase for determining the final decision is used to confirm results with the group. 
Fig. 1 Decision-making phases. $D M$ decision-making

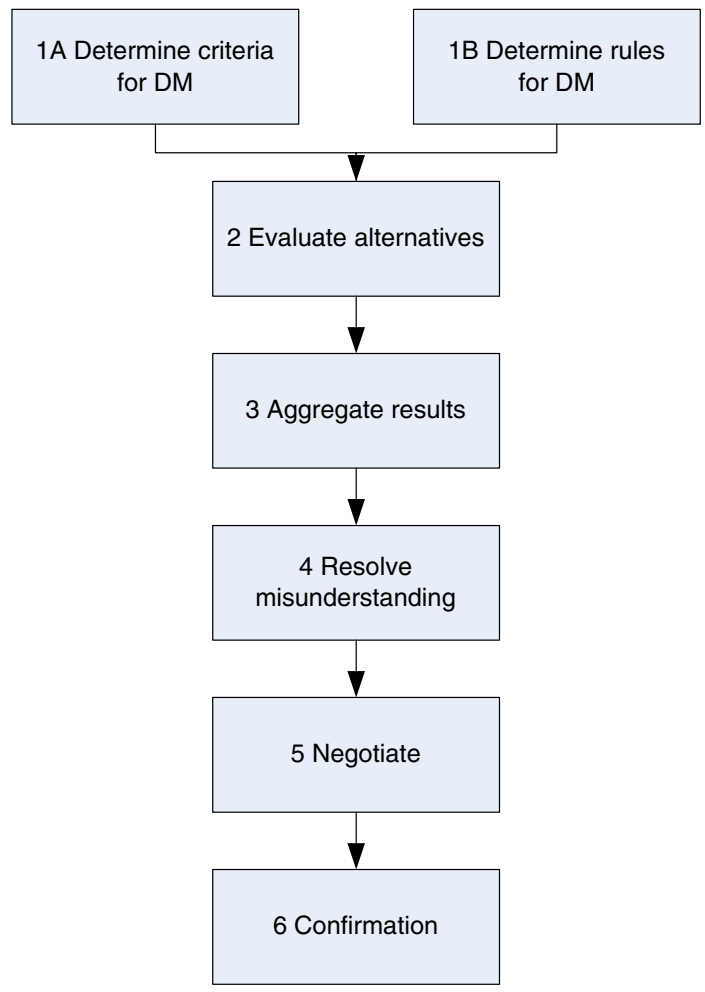

\section{Cognitive load in decision-making}

ThinkLets capture best practices in patterns of collaboration (Briggs et al. 2003; Kolfschoten et al. 2006; Vreede et al. 2006). They are highly detailed scripts that prescribe how to facilitate a collaborative activity. While originally developed for Group Support Systems, ThinkLets have evolved to become tool and domain independent scripts for collaborative activities. To get a detailed overview of activities involved in the decision-making phase, this paper analyses eight thinkLets that describe the cognitive activities involved in decision-making: CheckMark, StrawPoll, CrowBar, Pin the Tail on the Donkey, Point-Counter-Point, Moodring, and BucketVote, as documented in (Briggs and Vreede 2001). These eight thinkLets cover a broad range of decision-making activities, while each prescribes a different technique, they can be combined to cover the scope of decision-making activities described above. This paper identifies the cognitive activities required for each of these thinkLets. Overlap of cognitive activities is identified and removed. Next, cognitive activities are grouped in the decision-making phases. The resulting set of cognitive activities is compared to the decision-making aspects found in the literature described above, to verify completeness of the decision-making activities. This completeness check also considers basic cognitive activities: understanding, deciding, recalling (from memory), memorizing (store in memory) and inhibiting 
(to push away distracting thoughts or stimuli) (Rock 2009). An overview of in total forty-two cognitive activities in collaborative decision-making is provided, which are then classified based on their effectiveness.

As described above, effectiveness of decision-making has two key factors: objective quality of the decision and subjective support for the decision. Objective quality is about the rigor in comparing alternatives to understand and consider their implications and choose the alternative that best meets the decision criteria. It relates to what is often called System 2 Thinking (Chaiken et al. 1989; Kahneman 2003), i.e. explicit structured and analytic thinking as opposed to the more subconscious, intuitive and heuristic System 1 thinking. In our approach we are looking at processes that drive mainly towards System 2 thinking. Subjective support is about the accommodation of stakes and preferences and ensuring buy-in and commitment towards the outcome of the decision-making, a softer process but one that is essential to effective decision-making. This distinction between rational, objective decisions and social decision-making is extensively discussed in decision science. The rational choice perspective focuses on conscious analysis of costs, benefits, risks and outcomes, e.g. based on Expected Utility Theory (Schoemaker 1982). While the rational choice literature focuses on optimal choice, Simon posits that people are not able to explore or understand all consequences of a choice (Simon 1997). They propose as an alternative procedural rationality, a more subjective approach to rational decision-making (Simon 1997). Rational approaches tend to focus on decision support with tools and methods for reasoning, e.g. analysis (Belton and Stewart 2002), and modeling and simulation (Simon 1997). Social choice theory emphasizes a fair aggregation of preferences from stakeholders. From this viewpoint, exchanges of perspectives, fairness, unanimity and egalitarianism are important. From a social choice perspective, consensus is about finding a solution that is acceptable to all (Balthazard et al. 1998). For problem structuring, a combination of both is essential, as discussed in the characteristics above.

The overview of the cognitive activities involved in decision-making below is organized in the six phases of decision-making described above. Cognitive activities and their cognitive implication are listed in the table below, and numbered in the text with numbers between brackets ( ). Table 1 lists the numbered cognitive activities distinguished from the eight Evaluation ThinkLets, and our experience with these ThinkLets in practice.

\section{Cognitive activities in decision-making}

\subsection{Phase 1: decision preparation}

In the preparation phase a group receives (listens, reads) a specific decision-making task (1), a set of criteria for decision-making, and one or more decision-making rules. These criteria and rules could be given, or they can be created by the group through a separate process of divergence, convergence and decision-making, parallel to the main problem structuring process. The participants process these (2-4) for understanding, and memorizing, as they need to be maintained in working memory, especially the 
Table 1 Cognitive activities in collaborative decision-making

\begin{tabular}{llllll}
\hline Cognitive activity & RC & DQ & DS & $P$ & IE
\end{tabular}

Preparation objective, rules and criteria

2 Understand the decision task

3 Understand the decision criteria

4 Understanding the decision rules

5 Listening to the explanation of decision tool and method

6 Study/observe/try out the decision tool and method

7 Understanding one's task based on the decision tool and method

8 Cognitive effort to make the transition from understanding the decision-making task to performing it

Evaluation

9 Assess alternatives based on criteria

10 Consider implications of individual score

11 Compare scores for alternatives to consider strategic implications of ranking

12 Compare scores for alternatives to consider consistency of scoring

13 Compare scores for alternatives to consider relative weight of criteria

14 Mark/capture/write down evaluation or score

$\begin{array}{lllll}6 & 4 & 2 & 4 & \\ 3 & 2 & 1 & 1 & 1 \\ 4 & 2 & 4 & 2 & \\ 3 & 3 & 4 & 2 \\ 3 & 3 & 3 & 2 \\ 6 & 4 & 1 & 5\end{array}$

\section{Aggregation}

15 Understand group results (scores and ranking)

16 Understand the aggregation rule (how individual scores are combined into a group score)

17 Consider implications of the group score

18 Compare group score with personal score

19 Understand the aggregation rule for ranking (how the group scores are combined to become a ranking of alternatives)

20 Consider the implications of the ranking

21 Compare the group ranking with personal ranking

Resolving misunderstanding

22 Identify group disagreements

23 Assess the root cause of disagreement

24 Share or explain an evaluation perspective

25 Listen to other evaluation perspectives

26 Understand differences between perspectives

27 Reconsider scores in light of new understanding

28 Rephrase alternative to capture shared meaning

$\begin{array}{llll}7 & 7 & 3 & 1\end{array}$

$6 \quad 1 \quad 3 \quad 1$

$5 \quad 0 \quad 1 \quad 5$

$32 \quad 2 \quad 1 \quad 2$

$\begin{array}{llll}5 & 1 & 1 & 5\end{array}$

5

\section{4}

1

51

2

5

21

1


Table 1 continued

\begin{tabular}{lllllll}
\hline Cognitive activity & RC & DQ & DS & P & IE \\
\hline
\end{tabular}

Negotiation

29 Argue for specific evaluation

30 Listen to other arguments of others

31 Compare arguments with personal stakes

32 Consider compromise

33 Articulate proposal for compromise

$\begin{array}{lllll}\mathbf{7} & \mathbf{5} & \mathbf{5} & 2 & \\ \mathbf{7} & \mathbf{5} & 2 & 2 & 1 \\ \mathbf{7} & 3 & 3 & 2 & 2 \\ \mathbf{7} & \mathbf{5} & 2 & 3 & \\ \mathbf{6} & 2 & \mathbf{5} & 2 & \end{array}$

Confirmation

34 Consider decision outcome acceptance

35 Consider decision process fairness

$\begin{array}{llll}4 & 4 & 3 & 1\end{array}$

36 Consider trust in other stakeholders to commit to the decision outcome

37 Consider costs and success chance of further evaluation and negotiation

38 Understand final decision outcome

39 Assert commitment (yes or no)

Distraction

40 Consider new idea sparked by evaluation

41 Personal distraction

42 External distraction

$\begin{array}{lllll}6 & 7 & 2 & 1 & 1 \\ \mathbf{6} & 1 & 1 & 0 & 3 \\ \mathbf{6} & 1 & 1 & 0 & 3\end{array}$

The number of experts is indicated out of 7 experts that responded in total. Scores of $=>4$ experts are indicated in bold

$R C$ recognition, $D Q$ decision quality, $D S$ decision support, $P$ process, $I E$ ineffective

objective and criteria. Further, they receive instruction about the tools and methods that are to be used for the purpose of decision-making (5), observe, study or try them (6) and understand them to infer their personal task (7). Finally, a group makes the transition from preparation to the actual decision-making activity (8).

\subsection{Phase 2: evaluation}

In this phase, contributions from the brainstorming and convergence phase are evaluated based on the criteria presented in the preparation. To enable this evaluation, participants explore and understand implications of the alternatives on the systems they affect in the convergence phase (Kolfschoten and Brazier 2012). Decision support tools and models can be used to assess this impact, and are outside the scope of this paper. Based on the understanding of each alternative obtained in the convergence phase, participants now assess each alternative on each criterion (9), they consider implications of their score (10) and they compare scores for alternatives to consider strategic implications (how others score, how to use the evaluation system to manipulate the outcome) (11) and/or consistency of their 
scoring with respect to a single criterion (12), and with respect to the relative weight of criteria (13). Finally they mark their evaluation on a ballot either as a score, choice or qualitative evaluation (14).

\subsection{Phase 3: aggregation}

Results are aggregated according to the decision-making rules by a system or through means of shared representation. Depending on the method of evaluation an overview of the results is presented to the group, including aggregated group scores and rankings of alternatives. Participants interpret the group results (15). Participants comprehend the aggregation rule (16). They consider the implications of the group score (17) and compare these to their own evaluations to identify differences (18). Next, participants comprehend the way the ranking is created (19). They also consider the implications of the ranking of alternatives as a result of the group scores (20), and compare this ranking to their own ranking (21). In a qualitative evaluation, some of these aspects are much more difficult to understand, or they may be absent (e.g. no ranking, or no real group score, ideas that are more or less positively evaluated). However, similar considerations apply, and some of these aggregations may be created in the next phases rather than directly based on the initial evaluation.

\subsection{Phase 4: resolve misunderstanding}

Alternatives that receive highly divergent scores may indicate some type of disagreement. Briggs et al. (2005) distinguish five types of disagreement: different meaning (language), information asymmetry, different mental models (of understanding cause and effect), different goals (stakes, values, objectives) and different taste (personal preference). Furthermore, Briggs et al. assume these disagreements reflect on the outcome, its implication, and the likelihood of both outcome manifestation and implication manifestation. This categorization can be used to understand the source of disagreement; it can be some level of misunderstanding, or there can be a real conflict of stakes behind the disagreement. If a disagreement is mainly rooted in misunderstanding (of meaning, information asymmetry and mental models) it can be 'resolved' by exchanging and explaining different perspectives and understandings of the alternatives. Participants in this process identify disagreements (22), assess the source of disagreement (23) share/explain their perspective (24), listen to another perspective (25), understand another perspective and the differences between perspectives (26), and reconsider their evaluation (27). In some cases an alternative might be reformulated to reflect shared meaning (28). When misunderstanding is (partly) resolved, participants mark their new evaluations, looping back to the evaluation phase, or they brainstorm about reformulation to loop back to the brainstorming phase, which is outside the scope of this paper.

\subsection{Phase 5: negotiation}

When disagreement is rooted in differences in stakes, a process is initiated that is aimed at either convincing others to change the priorities in their stakes/goals 
(debate) or to change the alternative to compromise (negotiate). In this process participants argue for a specific evaluation (29), listen to arguments of others (30) and compare arguments with their personal goals/stakes (31). They then make counterarguments, and circle in this process until they consider a compromising proposal (32), they articulate this proposal (33) and then loop to the evaluation process to collect renewed evaluations and/or understand new disagreements. Note that evaluations in these follow-up rounds might be less formal in nature, or could be a complete iteration of the phase.

\subsection{Phase 6: decision-making}

Finally, a group converges in this process to a group evaluation and ranking of alternatives. In this final stage, participants may reflect on the decision-making process to consider if they can accept the outcomes (34), determine if the decision process is fair (35) and if they trust that others will commit to the outcome of the decision process (36). Finally, participants may consider the cost-benefit ratio of further evaluation and negotiation (37). To end the decision-making process a facilitator or group leader articulates the decision outcome as a course of action. Participants then understand the decision outcome (38), consider it (34-37), and assert their commitment (yes or no) (39). This process can result in ending the decision process, or reiterating steps.

\subsection{Distraction}

Finally, across phases, participants may be distracted by ideas triggered from considering the alternatives (40), off-task internal distractions (41) and off-task external distractions (42).

\section{Method}

Seven experts in facilitation and decision-making validated the list of cognitive activities. Given a description of each of the activities, they indicated whether each of the cognitive activities was likely to occur in collaborative decision-making, and whether each of the activities was instrumental (effective) to decision quality (objective 1, rational quality assessment of alternatives), decision support (objective 2 building agreement and commitment to the decision), or whether it is a procedural activity that is required for coordination, but does not contribute to the result ('extraneous'). Finally, they could classify activities as occurring but ineffective (e.g. distractions). The same experts are asked to indicate whether specific cognitive activities related to decision-making have been omitted.

The case description the experts received to explain the scope for the decisionmaking phase is the following:

"The group we imagine discusses the alternatives, and also has them on paper or on a whiteboard, so they can organize and structure them. They can also vote. When the group evaluates and compares alternatives, they can work in several steps 
towards a decision. These steps are organized in the experiment, but might in practice happen in parallel or in a different order. We assume that the group completes all cognitive tasks by themselves, and has a clear perception of the goal of the overall problem-solving task. They receive their instructions from a facilitator, but the facilitator does not help them with the content of the task. Note that we demarcate the preceding process of brainstorming, organizing and further defining alternatives."

\section{Results and interpretation}

Most of the seven experts recognized all but two of the cognitive activities listed, (namely considering fairness and considering the cost and success chance of further evaluation and negotiation). The classification of cognitive activities was less conclusive. Often multiple objectives are considered to be served by a single cognitive activity, by a majority of the seven experts. The bold numbers are classifications, where a majority of the experts agreed on one classification. The results of this limited explorative sample are described below in detail for each phase.

\subsection{Phase 1: preparation}

All preparation tasks were recognized by most of the experts. Experts indicated that understanding the task and criteria contributed to decision quality. For instance, decision criteria influence decision quality as they trigger more deliberate consideration of alternatives. Listening to the explanation of the task and tools are considered to be procedural activities.

\subsection{Phase 2: evaluation}

Evaluation activities seem to contribute to the objectives of decision-making, but the experts do not seem to agree to which of these objectives the activities contribute directly. Also, not all evaluation tasks are considered likely to occur, especially the comparison and implication reflection activities might occur, but are not recognized by all.

\subsection{Phase 3: aggregation}

While all these activities are recognized by a majority of the experts, similar to the evaluation task, few activities in this task can be classified to contribute to only one objective. Understanding the results and the aggregation rule seems to benefit decision support.

\subsection{Phase 4: resolve misunderstanding}

All activities in this phase are recognized by a majority of the experts. Resolving misunderstanding again seems to facilitate both decision quality and decision 
support. However, a focus here seems to be on quality. As resolving misunderstanding is the rational approach to decision-making, and negotiation reflects more the social process, this emphasis seems logical. However, several activities also score on facilitating decision support as such support is volatile when based on misunderstanding.

\subsection{Phase 5: negotiation}

Negotiation activities are also recognized by the group. Negotiation activities have been classified to also contribute to both decision objectives. As negotiation can address both the outcomes of decision-making as well as the way the decision was made, this makes sense.

\subsection{Phase 6: confirmation}

Understanding the outcome, considering acceptance and asserting commitment are recognized activities. The other considerations are not recognized by a majority. One expert remarked that these should take place, but that (s)he deemed it not very likely that they would without specific facilitation focus. Classifications are not very strong, but understanding the outcome seems to support both quality and support.

\subsection{Distraction}

Distraction is recognized except where it concerns new ideas, which are considered to support decision quality.

\section{Cognitive activities in collaborative problem structuring}

The cognitive activities in decision-making identified in this paper overlap with the types of cognitive load identified in the preceding phases of divergence and convergence (Kolfschoten 2011; Kolfschoten and Brazier 2012). Using similar expert validations, for divergence we found forty-seven cognitive activities and for convergence we found forty-two cognitive activities. When considering the total overview of cognitive activities, we can observe patterns in the sources of cognitive load, which are different than the three types of cognitive load in CLT (Sweller 1988). Below these types of cognitive load are summarized as eight sources of cognitive load in Problem Structuring. For each source examples of the decisionmaking phase above, and of the divergence and convergence process published earlier are provided.

1. Information processing load.

This is the inherent cognitive load associated to the complexity of the task, similar to the intrinsic cognitive load distinguished in CLT (Sweller 1988). The 
level depends on the expertise of the participant. In decision-making, the cognitive load associated with assessment and understanding the ranking are examples of direct processing and construction of new information. In brainstorming, creativity is a direct information processing task, and in convergence, classification is such task.

\section{Communication load.}

This is the cognitive load associated with reading, listening, formulation of perspectives and explication of this formulation, for example, in the preparation phase, where the task is communicated. In decision-making, marking the score is an example of such task. Communication load is unique to collaboration, as CLT is focused on individual learning. However, it could be considered as extraneous load, as communication activities are mostly scored by experts are procedural.

\section{Coordination load.}

This is the cognitive load associated with distributing tasks and inferring and understanding a participant's own task in the context of the overall problem structuring process. Understanding a participant's own task is a cognitive activity that recurs in all preparation phases. Similar to communication load, this cognitive load is procedural, and therefore extraneous. It is also unique to collaboration.

\section{Personal reflection load.}

This is the cognitive load of considering implications of the things a participant learns during the problem structuring process, and the things that a participant considers to bring into the problem structuring process. In decision-making, the group score and ranking are results that could lead to personal reflection as the choices made by the group can have an impact on one's tasks and role. In brainstorming and convergence, similar reflection on outcomes is listed. These reflections might occur also in learning, but are not directly distinguished in CLT, as they typically go beyond the learning task.

\section{Strategic reflection load.}

This is the cognitive load of considering the strategic and political implications of things a participant learns during the problem structuring process, and the things that a participant considers to bring into the problem structuring process. In decision-making activities such as acceptance and commitment, are examples of strategic considerations. Also in brainstorming and convergence, such strategic reflections based on results are listed. CLT has no separate cognitive load source describing strategic reflection, but it would be part of intrinsic cognitive load in 
complex, real world problem-solving tasks. The distinction of understanding a system as it is, and as it could be when alternative solutions are implemented is important to separate in collaborative model building (Barjis et al. 2009).

6. Quality reflection load.

This is the cognitive load of assessing the quality of the alternatives, perspectives, outcomes and the process of problem structuring. In decision-making fairness is a quality criterion, in brainstorming completeness is a quality criterion and in convergence consistency is a reflection criterion. Quality reflection could be a type of germane load, as it affects the quality (completeness, consistency) of schema's developed in long-term memory.

\section{Empathizing load.}

This is the cognitive load of stepping into the role and perspective of another stakeholder to look at the problem from a different perspective, and to understand the consequences of choices and decisions for others. In decision-making activities such as understanding another perspective were identified. In brainstorming and convergence, these tasks also occur when different ideas and structures are presented. Empathizing load is important to stimulate in problem structuring methods, and is again unique to collaborative tasks.

\section{Distraction load.}

This is the cognitive load associated with distractions, both external as well as thoughts that are triggered by the problem-solving process, but that are not relevant for the task. In all activity overviews, distraction was acknowledged as a cognitive activity that occurs. Distraction also occurs in learning, and is probably covered under extraneous load. Likewise, it should be reduced. Given that problem structuring activities stimulate thinking in different perspectives, the chance of 'triggering' a distracting train of thought is perhaps more likely.

\section{Discussion}

This aggregated overview of eight sources of cognitive load in collaborative problem structuring can offer an interesting framework for the analysis of problem structuring methods. Given the six characteristics of problem structuring methods, we now reflect on the insights we can gain from a cognitive perspective on these methods. We will reflect on how different sources of cognitive load play a role in the characteristics of problem structuring methods, and give an example of how these manifest in decision-making in particular. 
The use of one or more models as transactional objects helps to structure the information the group shares in the problem structuring effort, thus facilitating information processing (1). Information processing is strongly facilitated by visualization though a model. A model helps to capture the interdependencies of some of the factors that constitute the problem, and therewith to systematically detect misunderstanding and differences in perspectives that obstruct shared understanding and agreement. Further, the modeling rules help the group to perform quality assessments such as consistency checks (6). In decision-making a typical model used is a score card or multi-criteria model. Such a model offers the group an overview of alternatives and their value in light of different criteria.

The objective of increasing the overall productivity of the group process can be explained by understanding how facilitators direct attention by splitting the problem analysis task in several steps [reducing coordination load (3)], which helps to avoid cognitive overload. Also, facilitators can support the group to reduce distraction, and to minimize the cognitive load spend on communication and coordination (2, 3 ), by offering the group clear and precise instructions. In decision-making communication and coordination losses are most common in misunderstandings when either criteria or alternatives are not clearly defined, or not properly explained. Such misunderstanding can cause divergence in evaluation results which can in turn be wrongfully judged as fundamental disagreements. Facilitators should guard that the group spends sufficient time to build shared understanding on the alternatives and criteria.

Attention to facilitation of an effective participative group process is ensuring active participation of all stakeholders, which is important in a high cognitive load task, as reduced attention might cause people to 'loose track' of what is going on in the process. To create a state of high engagement or 'flow'(Czikszentmihalyi 1990) it is important that participants are goal focused. Further, facilitators should help the group to balance cognitive load, to help the group focusing attention on the task, and to maintain a link between different steps in the task and the 'big picture' to reduce coordination load (3). Further, a state of flow helps people to inhibit distracting thought processes (8). In decision-making the key instrument to link the steps is the set of rules for decision-making. These rules should be defined, clarified and understood before the evaluation. Changing the rules during the process can cause participants to loose trust and withdraw commitment to the results.

The significance of facilitation skills in effective model building suggests that facilitation is the main way in which cognitive attention is focused on different task aspects. Facilitation of problem structuring methods helps in minimizing the cognitive load of communication and coordination $(2,3)$, and maximizing the cognitive capacity available for information processing (1) and quality reflection (6). Effective modelling helps the group to create a systematic, complete and consistent overview of a system. Such an overview is an important basis for schema building and stimulates the understanding of the principles and mechanisms underlying a system as patterns (Kolfschoten et al. 2010). In decision-making, consistency and completeness need to be guarded in the voting criteria and scales to enable rigorous evaluation of alternatives. 
A focus on creating agreement and closure rather than solutions, suggests that problem structuring methods need to pay active attention to the reflective sources of cognitive load such as strategic (5), personal, (4) and empathic (7) reflection. These reflection steps help the group to understand different perspectives, which is an important basis to find solutions that all participants can commit to. The negotiation and confirmation phase are the key steps in decision-making that stimulate such reflection and help the group to assess their level of agreement and to resolve remaining points of disagreement.

A respect for multiple perspectives indicates that problem structuring methods help in capturing different perspectives, which supports reflection of the strategic (5) and personal (4) implications of the problem, as well as emphasizing (7) with a different perspective. These reflections help to build support and shared understanding of the final model and outcomes. However, it is unlikely that participants do not reflect earlier, and especially when they consider to bring new information or new perspectives into the problem-solving process. Problem structuring methods help to create an environment in which barriers to share different perspectives are lowered. In the decision-making phase, the group is further stimulated to make explicit commitments and agreements. This phase will reveal hidden perspectives when they did not surface before. Explicit evaluation of alternatives on criteria will reveal to the group to what extent they reached consensus and where disagreements remain.

Finally, problem structuring methods support an alternation between analysis of judgmental inputs and the application of judgment of analytic outputs. Judgment triggers reflection, and encouraging different types of reflections $(4,5,6,7)$ can help to increase support and buy-in for the end products of the problem-solving effort. Facilitators support this process by challenging hidden assumptions and resolving misunderstanding. In the decision-making phase, this is particularly achieved if the group is able to compare preference [support, commitment $(4,5,7)$ ] with objective judgments [analytical outputs (6)]. This is achieved when explicit evaluations are combined with negotiation techniques.

\section{Conclusions}

Problem structuring is a process that consists of analysis and structuring of a problem to derive alternative solutions, and ultimately to choose a course of action to solve the problem. Problem structuring methods tend to focus on the first two phases, but some techniques such as SODA and JOURNEY (Eden and Ackermann 2004) also describe a final phase with negotiation and building agreement; the phase of decision-making this paper addresses. This paper offers a first framework and classification of steps in a decision-making process from a cognitive load perspective. Previous work explores this perspective for divergence (brainstorming) (Kolfschoten 2011) and convergence (information analysis and structuration) (Kolfschoten and Brazier 2012). This paper adds a cognitive perspective on decision-making to understand the cognitive load involved in evaluation and consensus building to reach an agreement among stakeholders. The discussion 
compares the three phases of problem structuration, to derive first patterns in the cognitive load involved in these processes.

Based on an initial comparison of the three sets of cognitive activities, eight sources of cognitive load in collaboration are identified. The results of this study can be used as a basis to design more effective problem structuring methods, models and processes, taking into account the cognitive effort involved in these tasks. It can also be used to understand why the current practices work effectively in facilitating shared understanding, mutual learning and rigorous analysis of alternatives to reach agreement, and how they can be further improved. Practitioners in problem structuring methods can use this overview of sources of cognitive load, as well as the three more detailed overviews of cognitive activities involved in problem structuration, to analyze the sources of cognitive load in their problem structuration process, and to deliberately balance cognitive load in these processes to further facilitate effective problem structuration.

The presented study has limitations in the sense that the list of eight sources of cognitive load is not yet validated. A potential ninth source of cognitive load is for instance the cognitive load associated with emotions related to the problem-solving task. Next, the classification of cognitive activities is, like in the brainstorming and convergence phase, not very conclusive. It could very well be that the elementary level of cognitive tasks that allows us to classify how they contribute to the outcome of the cognitive task, has not yet been found, and that we need to decompose the list in yet smaller cognitive steps. Alternatively, it could be that this level of abstraction is the instrumental level, as the cognitive activities in collaborative problem structuring generally focus on multiple outcomes, such as in decision-making both decision quality and preference or decision support. This requires an abstraction level that allows for this type of ambiguity. Finally, the results in this study are highly qualitative, and evaluation was done by only seven experts. It might be possible to use for instance eye tracking or transcript analysis to perform a more quantitative evaluation of these cognitive activities, and to enable analysis of the amount of cognitive load that is imposed by different sources over time. These insights would enable us to develop a cognitive theory of collaboration that explains how cognitive load is balanced effectively. Such theory would be an important basis to further understand the mechanisms that help people to cope up with the high cognitive load of collaborative problem-solving activities. Understanding these coping mechanisms will help to improve existing and develop new, more effective support methods and tools to stimulate productive collaboration in problem structuring. Furthermore, such theoretical framework, once properly validated, would offer a basis for a more objective and precise measurement and evaluation of the effectiveness of problem structuring methods.

\section{References}

Ackoff RL (1978) The art of problem solving. Wiley

Balthazard P, Ferrell WR, Aguilar DL (1998) Influence allocation methods in group decision support systems. Group Decis Negot 7(4):347-362 
Barjis J, Kolfschoten GL, Verbraeck A (2009) Collaborative enterprise modeling. In Proper E, Harmsen F, Dietz JLG (eds) Advances in enterprise engineering II. vol. LNBIP 28. Springer, Berlin, pp 50-62

Belton V, Stewart TJ (2002) Multiple criteria decision analysis, an integrated approach. Kluwer, Dordrecht

Brady RH (1967) Computers in top-level decision making. Harvard Business Review, pp 67-76

Bray DA (2008) Information pollution, knowledge overload, limited attention spans, and our responsibilities as IS professionals. Paper presented at the GITMA World Conference Atlanta

Briggs RO, de Vreede GJ (2001) ThinkLets, building blocks for concerted collaboration. Delft, Delft University of Technology

Briggs RO, de Vreede GJ, Nunamaker JF Jr (2003) Collaboration engineering with thinklets to pursue sustained success with group support systems. J Manag Inf Syst 19(4):31-63

Briggs RO, Kolfschoten GL, de Vreede GJ, (2005). Toward a theoretical model of consensus building. Paper presented at the Americas Conference on Information Systems, Omaha

Briggs RO, Kolfschoten GL, de Vreede GJ, Albrecht CC, Lukosch SG, Dean DL (2009) A seven-layer model of collaboration: separation of concerns for designers of collaboration systems. Paper presented at the international conference on information systems

Chaiken S, Liberman A, Eagly AH (1989) Heuristic and systematic information processing within and beyond the persuasion context. In: Uleman JS, Bargh JA (eds) Unintended Thought. Guilford, New York, pp 212-252

Checkland PB (1981) Systems thinking, systems practice. Wiley, Chichester

Couger JD (1995) Creative problem solving and opportunity finding. Boyd And Fraser, Danvers

Czikszentmihalyi M (1990) Flow. The psychology of optimal experience. Harper Perennial, New York

de Vreede GJ, Briggs RO, Kolfschoten GL (2006) ThinkLets: a pattern language for facilitated and practitioner-guided collaboration processes. Int J Comput Appl Technol 25(2/3):140-154

Dillenbourg P, Betrancourt M (2006) Collaboration load. In Elen J Clark RE (eds) Handling complexity in learning environments: Research and theory. Pergamon, pp 142-163

Drucker PF (1967) The effective executive. London

Eppler JM, Mengis J (2004) The concept of information overload: a review of literature from organizational science, accounting, marketing, mis and related diciplines. Inf Soc 20(5):325-344

Farrington J (2011) From the research: myths worth dispelling: seven plus or minus two. Perform Improv Q 23(4):113-116

Fitousi D, Wenger MJ (2011) Processing capacity under perceptual and cognitive load: a closer look at load theory. J Exp Psychol Hum Percept Perform 37(3):781-798

Hackman JR, Morris CG (1975) Group tasks, group interaction process, and group performance effectiveness: a review and proposed integration. In: Berkowitz L (ed) Advances in experimental social psychology, vol 8. Academic Press, New York

Kahneman D (2003) Maps of bounded rationality: psychology for behavioral economics. Am Econ Rev 93(5):1449-1475

Kalyuga S, Ayres P, Chandler P, Sweller J (2003) The expertise reversal effect. Edu Psychol 38(1):23-31

Kolfschoten GL (2011) Cognitive load in collaboration-brainstorming. Paper presented at the Hawaii international conference on system science, Kauai, HI

Kolfschoten GL, Brazier F (2012) Cognitive load in collaboration-convergence. Paper presented at the Hawaii international conference on system science, Maui

Kolfschoten GL, Briggs RO, de Vreede GJ, Jacobs PHM, Appelman JH (2006) Conceptual foundation of the thinklet concept for collaboration engineering. Int J Human Comput Sci 64(7):611-621

Kolfschoten GL, Lukosch SG, Verbraeck A, Valentin E, de Vreede GJ (2010) Cognitive learning efficiency through the use of design patterns in teaching. Comput Edu 54(3):652-660

Miller GA (1956) The magical number seven plus or minus two: some limits on our capacity for processing information. Psychol Rev 63(2):81-97

Mitroff II, Betz F, Pondly LR, Sagasty F (1974) On managing science in the systems age: two schemas for the study of science as a whole systems phenomenon. TIMS Interf 4(3):46-58

Nijstad BA, Stroebe W, Lodewijkx HFM (2006) The illusion of group productivity: a Reduction of Failures Explanation. Eur J Soc Psychol 36:31-48

Paas FGWC, van Merrienboer JG (1994) Variability of worked examples and transfer of geometrical problem-solving sills: a cognitive load approach. J Edu Psychol 86(1):122-133

Paas F, Renkl A, Sweller J (2004) Cognitive load theory: instructional implications of the interaction between information structures and cognitive architecture. Instr Sci 32(1):1-8 
Pinsonneault A, Barki H, Gallupe B, Hoppen N (1999) Electronic brainstorming: the illusion of productivity. Inf Syst Res 10(2):110-133

Pollock E, Chandler P, Sweller J (2002) Assimilating complex information. Learn Instr 12(1):61-86

Reinig BA, Briggs RO, Nunamaker JF Jr (2007) On the measurement of ideation quality. J Manag Inf Syst 23(4):143-161

Rock D (2009) Your brain at work. HarperCollins Publishers, New York

Santanen EL (2005) Resolving ideation paradoxes: seeing apples as oranges through the clarity of thinklets. Paper presented at the Hawaii international conference on system sciences, Los Alamitos

Schoemaker P (1982) The expected utility model: its variants, purposes, evidence and limitations. J Econ Lit 20(2):529-563

Schwenk CR (1984) Simplification processes in strategic decision-making. Strateg Manag J 5(2):111-128

Simon HA (1960) The new science of management decision. Prentice Hall, New York

Simon HA (1973) The structure of Ill structured problems. Artif Intell 4:181-201

Simon HA (1974) How big is a chunk? Science 183(4124):482-488

Simon HA (1980) Cognitive science: the newest science of the artificial. Cogn Sci 4(1):33-46

Simon HA (1997) Rational decision making in business organizations. Am Econ Rev 69(4):493-513

Stroebe W, Nijstad BA, Rietzschel E (2010) Beyond productivity loss in brainstorming groups: the evolution of a question. Adv Exp Soc Psychol 43:157-203

Sweller J (1988) Cognitive load during problem solving: effects on learning. Cogn Sci 12(2):257-285

Sweller J, van Merrienboer JG, Paas FGWC (1998) Cognitive architecture and instructional design. Edu Psychol Rev 10(3):251-296

Valacich JS, Dennis AR, Connolly T (1994) Group versus individual brainstorming: a new ending to an old story. Organ Behav Hum Decis Process 57:448-467

Vennix Jac AM, Andersen David F, Richardson George P, Rohrbaugh John (1992) Model-building for group decision support: issues and alternatives in knowledge elicitation support. Eur J Oper Res 59:28-41 